\title{
Photocurrent Increase in Thin Film Solar Cells by Guided Mode Excitation
}

\author{
K. Söderström, J. Escarré, O. Cubero, F.-J. Haug and C. Ballif \\ Ecole Polytechnique Fédérale de Lausanne (EPFL), Institute of Microengineering (IMT), \\ Photovoltaics and Thin Film Electronics Laboratory, Rue Louis Breguet 2, 2000 Neuchâtel, Switzerland. \\ Corresponding author e-mail: karin.soederstroem@epfl.ch,
}

\begin{abstract}
Angle resolved measurements of the external quantum efficiency of a-Si solar cells deposited on a grating show strong absorption phenomena which are well explained with the guided mode structure in an equivalent flat multilayer system.

OCIS codes: (040.5350) Photovoltaic, (130.2790) Guided waves.
\end{abstract}

Thin film silicon solar cells require thin absorbing layers for high throughput considerations and better stability against light induced degradation which leads to a reduction of the electrical field inside the device. To achieve high efficiency in these devices, it is necessary to enhance the short circuit current density using light trapping. Light trapping by interface texturing has been proven to successfully increase the efficiency of solar cells in n-i-p and p-i-n configuration. This could be done by periodic [1-3] or random texturing [4-6]. However, the underlying mechanism of light trapping is not clearly understood yet as optical characterization is often done with far field measurements.

In this contribution, we would like to evidence one of the mechanisms of light trapping. We relate the absorption within a solar cell to the guided modes that propagates in a system of layers. As the absorbing silicon layer is embedded between two media having lower index of refraction, a thin film of amorphous silicon can be considered as a wave guiding medium, alas an absorbing one.

A sinusoidal grating substrate with a period $\mathrm{P}=830 \mathrm{~nm}$ and an amplitude $\mathrm{h}=70 \mathrm{~nm}$ (half the peak to valley depth) was made using an "in-house" nano-imprinting technique. A silver layer of $120 \mathrm{~nm}$ was deposited by sputtering on top of the grating as back contact (according to ref. [7] such a layer is sufficient to be considered as semi infinite in the subsequent calculation). $60 \mathrm{~nm}$ of $\mathrm{ZnO}$ was deposited by sputtering before the deposition of $235 \mathrm{~nm}$ of amorphous silicon using plasma enhanced chemical vapor deposition (PECVD). The front contact was made by sputtering of $65 \mathrm{~nm}$ of ITO which also serves as anti-reflective coating. P and S polarized angle resolved external quantum efficiency was measured, varying the angle of incidences between 0 and $55^{\circ}$ (measured with respect to the surface normal). Figure 1 shows strong absorption signatures in the weakly absorbing region between 1.6 and 2 $\mathrm{eV}$ whose energies vary systematically with the angle of incidence.

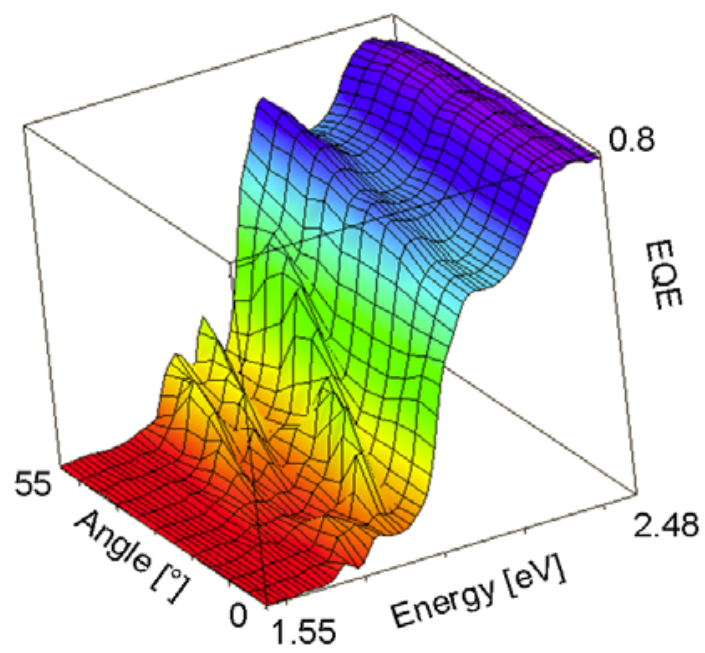

Figure 1: Angle resolved external efficiency of a solar cell on a sinusoidal grating with a period of $830 \mathrm{~nm}$. The incident light is P polarized (polarization parallel to grating groves), and the incident angle was varied between 0 and $55^{\circ}$.

We interpret the absorption phenomena as grating coupling between the wave vector component parallel to the surface $k_{/ /}^{i}$ and eigenmodes of the structure. The wave vectors of the guided modes for both, $\mathrm{P}$ and $\mathrm{S}$ polarization 


\section{PTuB6.pdf}

were estimated using a flat interface waveguide according to ref. [8]. The device was approximated as: semiinfinite silver / $60 \mathrm{~nm}$ of $\mathrm{ZnO} / 235 \mathrm{~nm}$ of amorphous silicon / 65nm of ITO. A photon impinging on a grating structure can gain or loose momentum equal to a multiple of the reciprocal lattice vector which yields a multitude of equivalent wave vectors $k^{\prime}$ given by:

$$
k^{\prime}=k_{/ /}^{i}+m \frac{2 \pi}{P}=\sin \theta^{i} * \frac{2 \pi}{\lambda^{i}}+m \frac{2 \pi}{P}
$$

where $\mathrm{k}_{/ /,}^{\mathrm{i}} \theta^{\mathrm{i}}, \lambda^{\mathrm{i}}$ are the incident parallel wave vector, angle and wavelength, respectively, $\mathrm{m}$ can be any integer and $\mathrm{P}$ is the period of the grating. Excitation occurs whenever the wave vector of an eigenmode propagating in the silicon layer is equal to $k^{\prime}$.

Thus a comparison between the $k$-vector calculations of the guided mode in the structure was made with the experimental values of the impinging $k$ vector, adding multiples of reciprocal grating vector, see figure 2. Still there is an ambiguity on the number of reciprocal vectors to add which can only be partly resolved by the systematic of the angular changes.

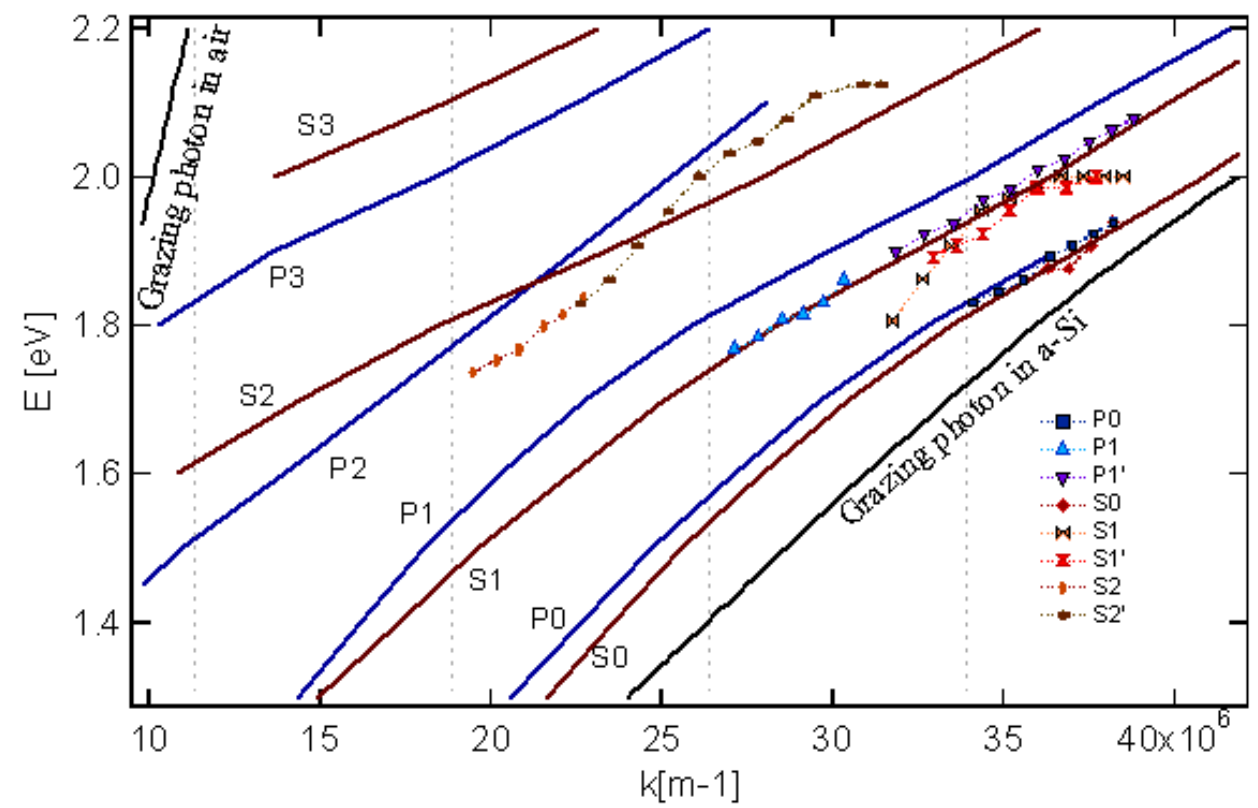

Figure 2: Dispersion diagram: Solid lines are extracted from calculations. Symbols are experimental data adding an integer number of vectors of the grating reciprocal lattice. The ' denotes experimental values attributed to the same mode but with a different number of vectors of the grating reciprocal lattice.

Figure 2 shows good correlation between experimental and theoretical values. It shows that the grating perturbation on the flat structure does not strongly alter the flat waveguide modes. The grating solely serves to couple the external electromagnetic field to confined propagating modes. These preliminary results indicate that the light trapping effect in thin film silicon solar cells corresponds to excitation of guided modes; however the flat interface model can not predict the coupling strength induced by the grating structure.

[1] H. Iida et al., "Efficiency of the a-Si:H solar cell and grain size of $\mathrm{SnO}_{2}$ transparent conductive film". Electron Device Letters, IEEE, Vol. 4, (1983), pp. 157-159.

[2] H. Sai and M. Kondo. "Effect of self-orderly textured back reflectors on light trapping in thin-film microcrystalline silicon solar cells". Journal of Applied Physics, Vol. 105 (2009), pp. 094511.

[3] T. Söderström et al., "TCOs for nip thin film silicon solar cells". Progress in Photovoltaics: Research and Applications, Vol. 17, (2009), pp. $165-176$.

[4] N. Senoussaoui et al., “Thin film solar cells with periodic coupler”, Thin Solid Films, Vol. 451-452, (2004), pp. 397-401.

[5] C. Haase et al., "Optical properties of thin-film silicon solar cells with grating couplers", Progress in photovoltaics: Research and Applicaitons, Vol. 14, (2006), pp. 629.

[6] H. Stiebig et al., "Silicon thin-film solar cells with rectangular-shaped grating couplers", Progress in Photovoltaics: Research and Applications, Vol. 14, (2006), pp. 13-24.

[7] R. Burns and H. Raether, "Plasma Resonance Radiation from Non Radiative Plasmons", Z. Physik, Vol. 237, 1970, pp. 98-106.

[8] F.-J. Haug et al., "Influence of the $\mathrm{ZnO}$ buffer on the guided mode structure in $\mathrm{Si} / \mathrm{ZnO} / \mathrm{Ag}$ multilayers", Journal of Applied Physics, Vol. 106, (2009), pp. 044502. 